\title{
A Method to Remotely Measure Amplitudes of Surface Vibrations with a Conventional Michelson Interferometer \\ Ralph Hohenstein ${ }^{1,2}$ Felix Tenner $^{1,2}$, Christian Brock ${ }^{1,2}$, Michael Schmidt ${ }^{1,2}$ \\ ${ }^{1}$ Institute of Photonic Technologies, University of Erlangen-Nuremberg, Paul-Gordan-Str.3, 91052 Erlangen, Germany, \\ ${ }^{2}$ Erlangen Graduate School in Advanced Optical Technologies, Paul-Gordan-Str.6, 91052 Erlangen, Germany \\ Ralph.Hohenstein@Ipt.uni-erlangen.de
}

\begin{abstract}
:
This paper introduces a method to measure the amplitude of surface vibrations using a conventional Michelson interferometer. Such amplitudes may be far greater than half of the used laser's wavelength. With relatively simple signal processing it is possible to measure amplitudes at a precision that is similar to or better than the precision of the fringe counting method (FCM) and that is hardly affected by the varying tilt an oscillating surface may exhibit during the reflection of the laser beam. The proposed method allows for monitoring amplitudes which proved to be too small to be reliably measured by our version of the FCM. Using a $532 \mathrm{~nm}$ laser and sampling rates of $192 \mathrm{kHz}$ amplitudes up to $133 \mu \mathrm{m}$ during vibrations at $79 \mathrm{~Hz}$ can be measured. The sensor is easy to construct and calibrate, much cheaper than conventional multi-wavelength interferometers and does not have the need for complex signal processing.
\end{abstract}

Key words: interferometry, discrete optical signal processing, spectrum, vibration, laser

\section{Introduction}

Fast, reliable and remote vibration measurement is an important tool for quality assurance for a lot of production processes. These demands can be met with a laser interferometer. Unfortunately, the working range of conventional Michelson interferometers is limited to amplitudes smaller than half the wavelength of the laser due to their measurement principle.

There are several efforts to enhance the measurement range. The most common technique is the fringe counting method (FCM). This technique allows for measuring amplitudes much larger than half the pilot laser's wavelength, by counting the fringes of the interferometric pattern. The higher the amplitude - the more maxima and minima are visible on the detector in each oscillation period. Unfortunately, this method is not suitable for measuring amplitudes that are low (below $\lambda / 2$ ) or that occur at high frequencies [1]. In these regions the signal-to-noise ratio decreases and it is more complicated to count closely spaced fringes. To fix this problem complex signal processing has to be done [2,3]. Other approaches use two lasers [4] to increase the detectable range of amplitudes which comes at the cost of higher technical complexity. Nevertheless it is possible to enlarge the working range of common FCM by taking several measurements and applying statistical data evaluation [5]. This method can be used for calibration, but is not fit for online measurements.

Another approach to enlarge the measurement range is the superheterodyne principle [6]. This method uses two frequency stabilized lasers with slightly different wavelengths. By supercomposing the beams a beat is formed with a long wavelength up to several meters. This increased wavelength allows the detection of high amplitudes, at the cost of reduced resolution of these measurements. Another disadvantage of this technique is the necessity to use two lasers and to stabilize both of them.

The fringe disappearance method shown by Pineda et. al. [7] is based on the use of a simple Michelson interferometer. The presented method, also known as "Bessel function minimum point method" requires the use of a bandpass filter tuned to the vibration frequency. The frequency has to be measured using some other method.

The method we introduce in this paper relies on applying a Michelson interferometer together with relatively simple signal processing. The interferometer is easy to build and calibrate. The method allows for measuring small as well as large vibration amplitudes that are well above the non-ambiguity range. Furthermore 
we observe that the system works in situations where the surface movement partially consists of tilting and multiple harmonics.

\section{Measurement Principle}

The mechanical and optical arrangement of the measurement system is that of a conventional Michelson interferometer (see Fig. 1). The measurement principle is based on the interferometric effect, where constructive or destructive interference between a reference and a phase shifted laser beam increases or decreases the measured overall intensity. Without movement of the illuminated surface the intensity of the interfered beams in a conventional Michelson interferometer remains static and so does the photodiode signal. A vibration of the reflective surface causes displacements which alter the phase difference between the two beams. This phase shift again alters the interference pattern on the photodiode. When monitoring the diode current related to the changing interference pattern in the time domain it seems that it is only possible to detect amplitudes less than half the pilot laser's wavelength $\lambda_{P}$, because the pattern on the photodiode ends up to have the same shape when the surface is displaced by an integer multiple of the wavelength.

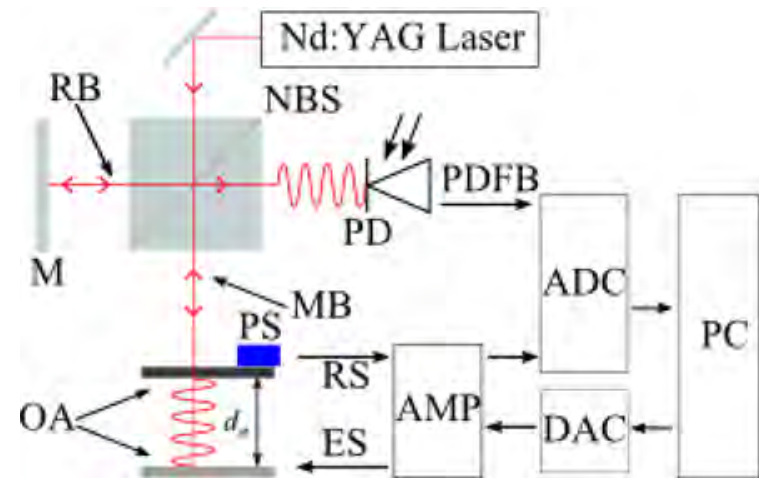

Fig. 1. Sketch of the experimental setup: M, Mirror; $N B S$, non-polarizing beam splitter; $R B$, reference beam; $M B$, measuring beam; $O A$, oscillating area; $P D$, photodiode; $P S$, piezoelectric sensor; $A D C, 24$ bit analogue-digital converter; DAC, 24 bit digitalanalogue converter; ES, excitation signal; RS, response signal; PDFB, photodiode feedback; AMP, signal amplifier

However, the dynamics of the photodiode signal get well influenced by how fast the interference pattern changes. Thus certain features of the dynamic spectrum of the photodiode signal may constitute invertible functions of the displacement amplitude. Invertibility here means that the displacement amplitude may be retrieved from the observation and analysis of such features. Imagine a surface displacement spanning $x$ wavelengths in one direction. Then the diode will be exposed $x$ times to the same pattern changes. The frequency of pattern changes scales with the amplitude or frequency of surface vibrations in a linear way. Thus there must be a significant frequency in the photodiode signal that is useful to estimate either the amplitude or the frequency of the main harmonic contained in the spectrum of surface vibrations. This idea will be detailed in the following.

\section{Analysis of the Sensor Signals}

When using an interferometer, two coherent beams (electromagnetic waves) are interfered. It can be assumed that the waves have the same propagation direction when detected by the photodiode. The intensity on the photodiode depends on the phase difference $\Delta \varphi$ between the reference and the reflected beam which is determined by the refractive index of the surrounding medium (e.g. $n_{0} \approx 1$ for air) and the path difference $\delta_{p}$ between those beams:

$\Delta \varphi=k \delta_{P}$, where $k=\frac{2 \pi n_{0}}{\lambda_{P}}$

In our case $\delta_{p}$ is given by the beam path length $\mathrm{d}_{0}$ for $\mathrm{t}=0$, a surface vibration with the unknown amplitude $d_{a}$ and the known frequency $f_{a}$ :

$\delta_{P}=d_{0}+d_{a} \sin \left(\omega_{o} t\right)$, where $\omega_{o}=2 \pi f_{a}$

With $I_{0}$ quantifying the intensity of noninterfering parts of the two beams falling onto the photodiode and $I_{A}$ quantifying the intensity of the interfering parts the intensity $I_{P}(t)$ on the detector over time can be written as [8]

$I_{P}(t)=I_{0}+I_{A} \cos (\Delta \varphi)$

with $n_{0} \approx 1$. The Fast Fourier Transformation (FFT) of Eq. 3 for different amplitudes $d_{a}$ and a frequency $f_{a}=79 \mathrm{~Hz}$ is shown in Fig. 2 .

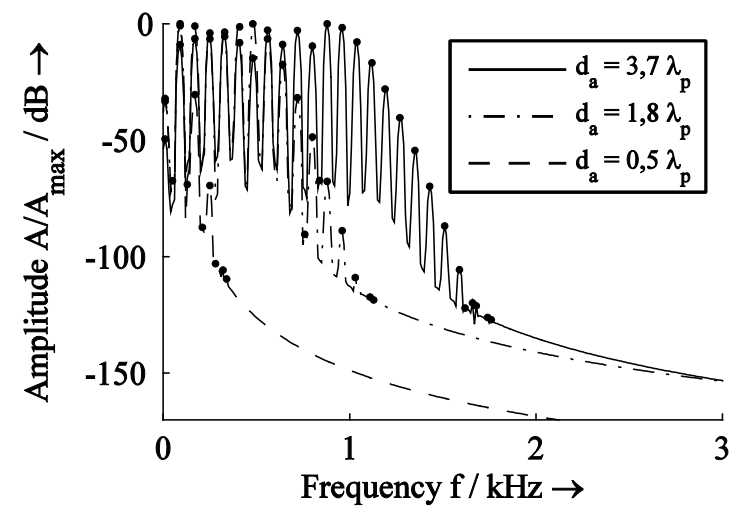

Fig. 2. Frequency spectrum of the modeled photodiode signals for different amplitudes $d_{a}$ (Eq. 3) at a constant frequency of $f_{a}=79 \mathrm{~Hz}$

The amplitudes were chosen to extend beyond half the pilot laser's wavelength, the limit of 
conventional interferometers. The diagram exhibits a direct relation between the vibration amplitude and the spectrum of the photodiode signal: The larger the vibration amplitude, the wider the spectrum of the photodiode signal.

To explain this behavior analytically, we calculate the Fourier coefficients $c_{k}$ of the Tperiodic intensity $I_{P}$ during harmonic vibrations:

$$
\begin{gathered}
I_{P}(t)=\lim _{n \rightarrow \infty} \sum_{k=-n}^{n} c_{k} e^{+j 2 \pi \frac{k}{T} t} \\
c_{k}=\frac{1}{T} \int_{0}^{T} I(t) e^{-j 2 \pi \frac{k}{T} t} d t
\end{gathered}
$$

Using the Dirac function $\delta$ and the Bessel function $J_{k}$ the Fourier coefficients $c_{k}$ can be calculated from:

$$
\begin{aligned}
& c_{k}=I_{0} \cdot \delta(k)+\frac{I_{A}}{2 \pi} \int_{0}^{2 \pi} \cos \left(x_{0}+x\right. \\
& \cdot \sin (\tau)) e^{-j k \tau} d \tau \\
& =I_{0} \cdot \delta(k)+I_{A} \cdot J_{k}(x) \cdot\left\{\begin{array}{lr}
\cos x_{0}, & k \text { even } \\
j \sin x_{0}, & k \text { odd }
\end{array}\right. \\
& \text { with } x_{0}=\frac{2 \pi n_{0} d_{0}}{\lambda} \text { and } x=\frac{2 \pi n_{0} d_{a}}{\lambda}
\end{aligned}
$$

When we are in the ideal situation, where $I_{0}$ is constant, Eq. 5 shows, that the spectrum of the photodiode signal is enveloped by a scaled Bessel function $J_{k}(x)$, where $x$ scales the amplitude $d_{a}$ of surface vibrations and where $k$ defines the locations $f=k / T=k \cdot f_{a}$ on the frequency axis of the spectrum. The periodicity of $I_{P}$ accompanies a discrete amplitude spectrum, which exhibits nonzero spikes at integer multiples of the frequency $f_{a}$ of harmonic surface vibrations. Bessel functions have a limit $k=x$ (approximately) above which these functions (and thus the spectrum of the photodiode signal) tend towards zero with rising $k$. This is the reason why the spectral width of the interference related part of the photodiode signal (represented by parameter $k$ ) quantifies the vibration amplitude (represented by parameter $x$ ) in a linear way. These facts are visible in Fig. 2.

\section{Measuring the Amplitude of the Surface Vibrations}

When fluctuations of $I_{0}$ remain low, it is possible to measure the absolute value of the amplitude of the surface vibrations by identifying the edge in the amplitude spectrum of the photodiode signal where the normalized amplitude $A / A_{\max }$ starts to decrease. We simulated different amplitudes and different frequencies using Matlab/Octave and Eq. 3. In each resulting spectrum we identified the location of the frequency peak which is closest to $f=0$ and of which the amplitude is damped by $-15 \mathrm{~dB}$ with respect to the maximum amplitude. The so defined edge frequency is in linear relation to the amplitude. Both, stronger amplitudes as well as higher frequencies of the surface vibration increase the edge frequency of the spectrum of the photodiode signal.

At the edge frequency $f_{\text {edge }}=k \cdot f_{a}$ we approximately have $k=x$. Therefore we can estimate

$f_{\text {edge }}=x \cdot f_{a}=\frac{2 \pi n_{0} d_{a}}{\lambda} f_{a}$

The maximum amplitude which can be measured with this method strongly depends on the realizable sampling frequency and the frequency of surface vibrations. Due to Shannon's theorem, $f_{\text {edge }}$ must not be larger than half the sampling frequency. Our $A D$ converter was sampling at $192 \mathrm{kHz}$. For a vibration at $f_{a}=79 \mathrm{~Hz}$ or $f_{a}=206 \mathrm{~Hz}$, the amplitudes $d_{a} / \lambda$ had to remain below $192 / 4 / \pi / 79=193$ or 74 respectively.

\section{Installation and Measurement}

For validating the simulations we conducted experiments using the setup shown in Fig. 1. There we applied a Roithner RLDD532-10-3 $\mathrm{Nd}: Y A G$ laser which operated at a wavelength of $532 \mathrm{~nm}$ and at a power output of $10 \mathrm{~mW}$. In order to generate surface vibrations at defined frequencies we attached a 60 Watt loudspeaker chassis capable of frequencies from 40 to $6500 \mathrm{~Hz}$ to a metal sheet. The surface of the sheet then oscillated with the loudspeaker membrane. To have sufficient amounts of laser light reflect back into the interferometer, we glued a mirror with better reflectivity to this vibrating surface and targeted the mirror by the interferometer's laser.

The loudspeaker's amplitude and frequency were set by a PC and an amplifier. Additionally a piezo sensor was attached to the metal sheet to obtain a reference measurement (see Fig. 1). The photodiode's signal was digitized by a 24 bit AD-converter at a sampling rate of $192 \mathrm{kHz}$.

\section{Results and Discussion}

Fig. 3a shows the signals of the piezo sensor and the photodiode for small amplitudes of the vibrating surface while the respective signals in Fig. $4 a$ were acquired during approximately six times larger amplitudes. The increasing amplitudes of the sinus which we enforced at the speaker input eventually added higher order harmonics to the piezo signal shown in Fig $4 a$ due to the higher total harmonic distortion of the loudspeaker for higher amplitudes. 
In Fig. $3 a$ the photo diode signal exhibits harmonic signal characteristics at the same frequency as the signal of the piezo sensor, which is $79 \mathrm{~Hz}$ in this case. This results from the small changes in the tilt of the mirror, causing a displacement of the measuring beam on the photodiode in every oscillation period, whereas the position of the reference beam on the photodiode remains constant.

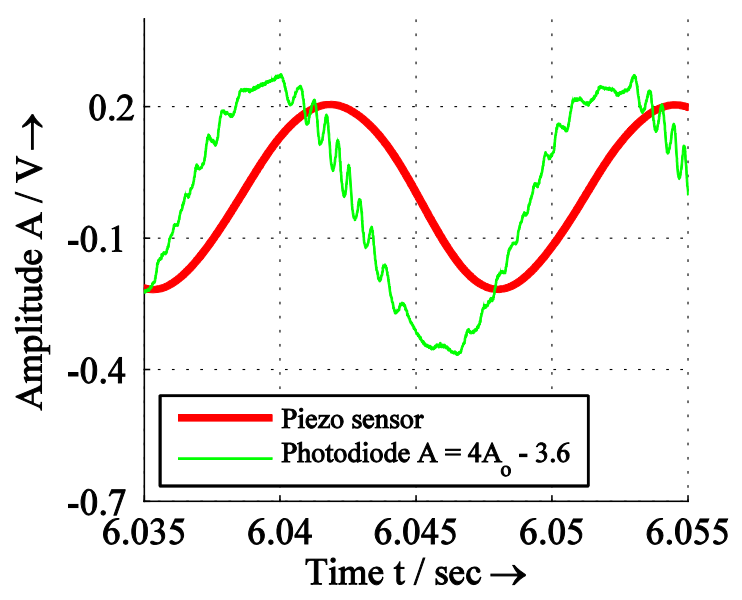

(a) Small vibration amplitude

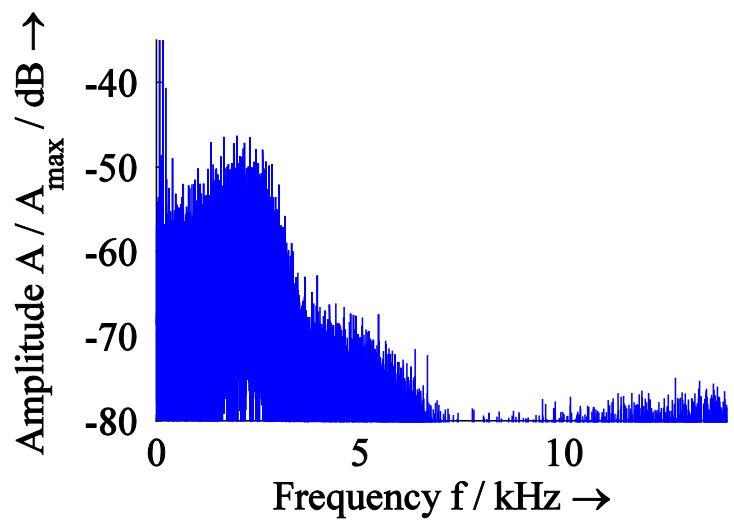

(b) Amplitude spectrum of photodiode signal for a small vibration amplitude

Fig. 3: Piezo sensor and photodiode signal during small amplitudes of the oscillating mirror surface (photodiode signal scaled, $A_{o}$ represents the original amplitude)

The interesting part of the diode signal is however the visible alternating changes in its modulation, see sections 1 and 2 in Fig. $4 a$. The diode signal's modulation in section 1 exhibits high frequencies whereas in section 2 it exhibits lower frequencies. The velocity of the oscillating surface is the lowest in the turning points of the piezo sensor's curve. Therefore the path difference changes more slowly, so that fewer maximums are visible in section 2 . The faster modulation in section 1 occurs always during high surface velocities. This constitutes the very idea that lead us to conduct these experiments. Higher frequency or larger amplitude of the vibration means faster surface displacement, means faster change in the interference pattern, means broader spectrum of the photodiode signal.

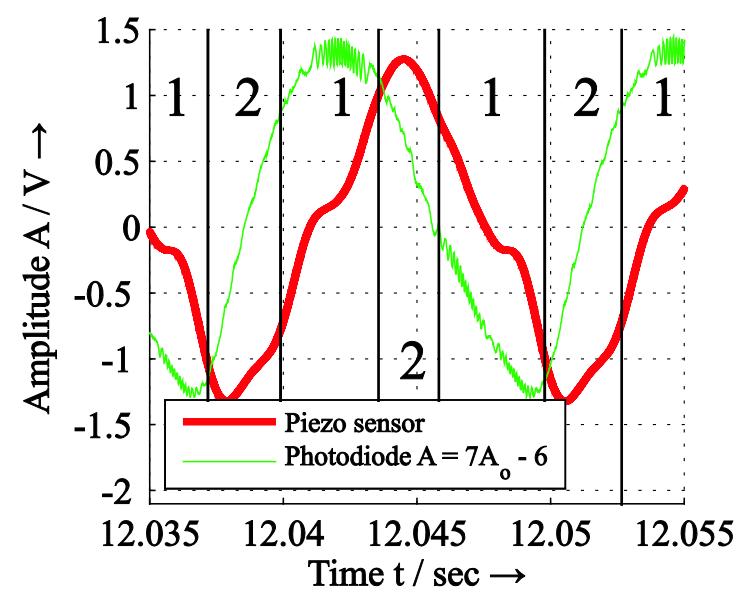

(a) Large vibration amplitude

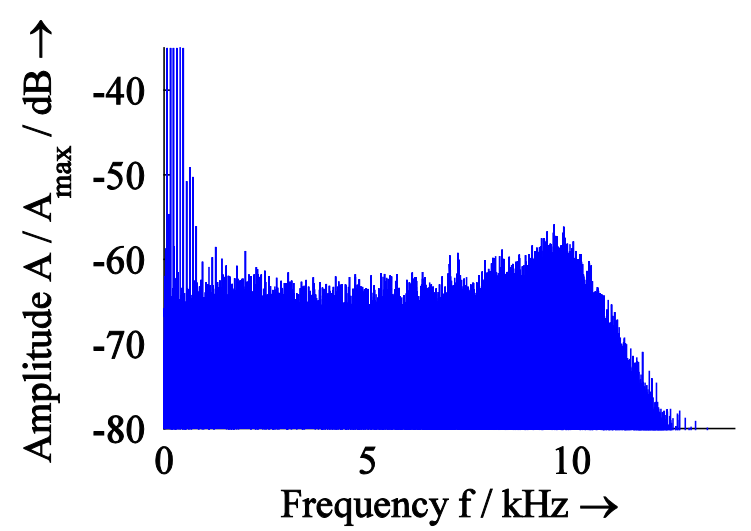

(b) Amplitude spectrum of photodiode signal for a large vibration amplitude

Fig. 4. Piezo sensor and photodiode signal during large amplitudes of the oscillating mirror surface (photodiode signal scaled, $A_{o}$ represents the original amplitude)

Our experiments thus confirmed this initial assumption as Fig. $3 \mathrm{~b}$ and $4 \mathrm{~b}$ show. In comparison with [7] the peaks in the resulting frequency spectra are denser due to the fact that the used frequency of surface vibrations is much lower in our experiments $(79 \mathrm{~Hz}$ to 4 $\mathrm{kHz}$ ). Due to electronic noise, surface tilt, nonzero vibration at higher harmonics and a nonzero gradient of the vibration amplitude over time our spectra contain non-integer multiples of the excitation frequency. However, the excitation frequency $f_{a}$ (first order harmonic of the vibration, here $79 \mathrm{~Hz}$ ) remained dominant in the photo diode spectrum. Thus calculation of $f_{a}$ from the photodiode spectrum was straight forward. Determination of $f_{a}$ then allowed us to restore the amplitude of surface vibrations from 
the frequency spectra, as described in the following.

Both signal sections in Fig. $3 a$ and $4 a$ were taken out of a longer time series shown in Fig. 5. The shown piezo signal indicates how the amplitude of the surface vibration varied over time. In a first step, we determined the amplitude using our version of the fringe counting method, which intends to extract fringes from underlying noise by calculating and analyzing the signal envelope.

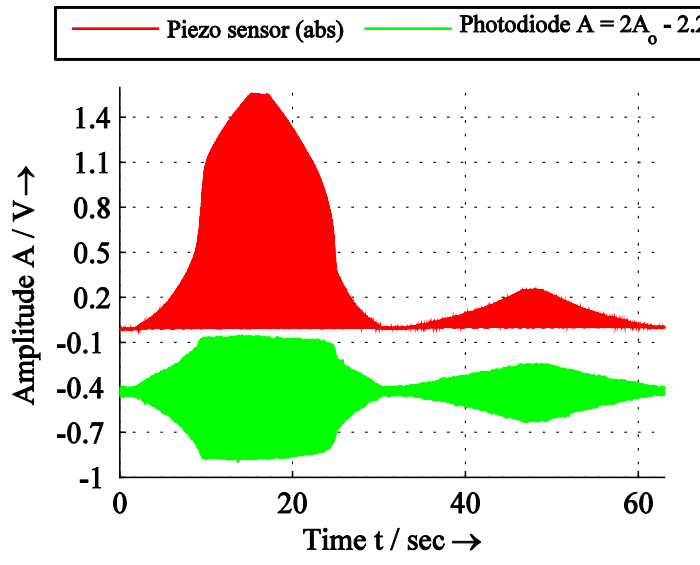

Fig. 5. Signal sequence of the measured photodiode's and piezo sensor's signal with constant frequency $f_{a}$ of $79 \mathrm{~Hz}$ and variation of the amplitude of the input signal to the loudspeaker; sample rate: $192 \mathrm{kHz}$; (green signal scaled, $A_{o}$ represents original amplitude)

Fig. 6a shows the result of our FCM, which we used for the measurement shown in Fig. 5 and which we generally refer to as "FCM" in the following. The results suggest that the FCM is only useful for large amplitudes during $t=16+/$ $6 s$. The error in the regions with lower amplitudes occurs due to the difficulty to count maxima in situations where the signal to noise ratio is low. Also a low pass filter with a cutoff frequency of $11 \mathrm{kHz}$, which was used in this analysis, did not improve the result significantly.

To track the specific edge frequency a running FFT was performed. The FFT was calculated for a subset of $\mathrm{N}$ samples around each sample of the photodiode signal in the time span (10 $22 \mathrm{sec}$ ) mentioned before, where $\mathrm{N}=96000$ for Fig. 6b. In each of the running spectra we determined the edge frequency by thresholding the envelope of the amplitude spectrum at $48 \mathrm{~dB}$ and by choosing the lowest frequency above which the envelope remains below the threshold. Using this edge frequency we estimated the vibration amplitude according to Eq. 7. The result is shown in Fig 6b.

Fig. 6b shows the result of our proposed $f_{\text {edge }}$ method for the complete time range. Differing from the output of our FCM algorithm shown in
Fig. 6a, we obtained strong correlations between the output of the $f_{\text {edge-method and the }}$ amplitude of the piezo signal even beyond the limits of our FCM algorithm, which suffers when amplitudes remain below $10 \lambda_{p}$.

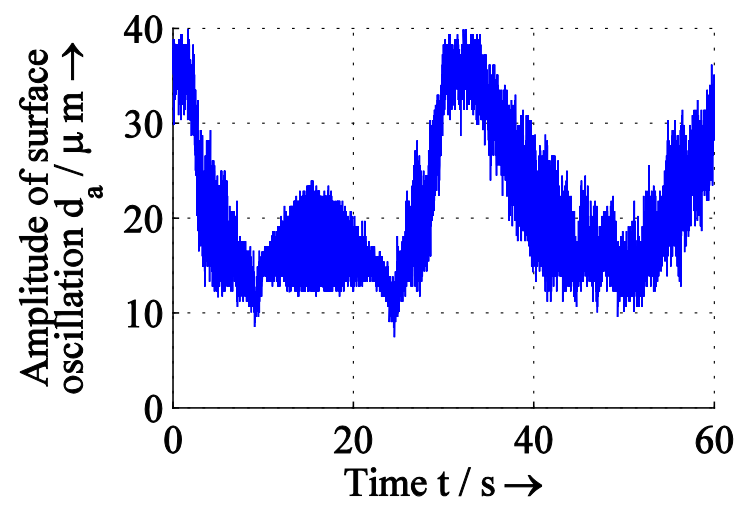

(a) Amplitude obtained using FCM

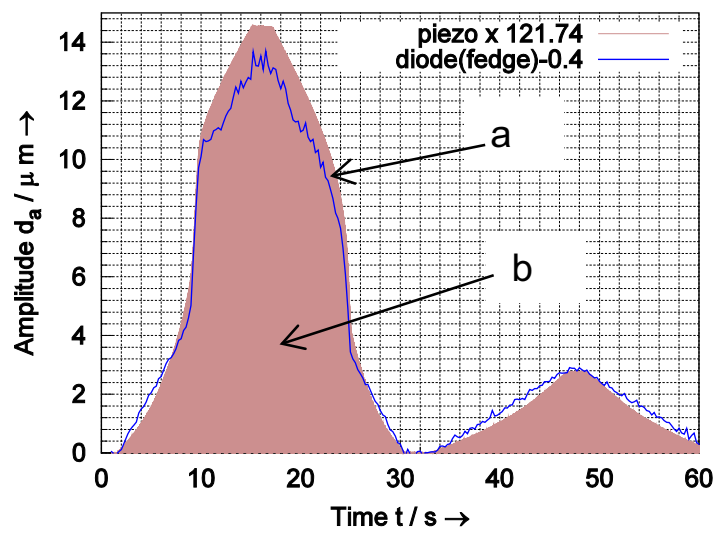

(b) Scaled upper half of piezo signal (curve b) and envelope obtained from photodiode signal using the $f_{\text {edge }}$-method (curve a)

Fig. 6. Calculated vibration amplitudes using the photodiode signal shown in Fig. 5

When using the $f_{\text {edge }}$-method the upper range of detectable amplitudes is only limited by available sampling rates as the photodiode signal has to be sampled at least at rate $f_{s}=$ $2 \cdot f_{\text {edge }}$, according to Shannon's Theorem. With a $532 \mathrm{~nm}$ laser wavelength the maximum measurable amplitude for $f_{\text {edge }}=96 \mathrm{kHz}$ is 133 $\mu \mathrm{m}$ at $f_{a}=79 \mathrm{~Hz}$ or $39 \mu \mathrm{m}$ at $f_{a}=206 \mathrm{~Hz}$. The amplitudes obtained from analyzing the photodiode signal slightly differ from the piezo signal envelope in Fig 6b, because our $f_{\text {edge- }}$ method tracks the first order harmonic while the piezo is affected by all higher order harmonics. Higher order harmonics occurred in our experiment at high amplitudes. Then the piezo signal envelope is the geometric sum of the amplitudes of all harmonics exceeding the result of the $f_{\text {edge }}$-based amplitude estimate slightly in Fig 6b. Note that the $f_{\text {edge }}$ based amplitude estimate is much less affected by 
noise than FCM. Because noise is spread over large regions of the photodiode's amplitude spectrum, the $f_{\text {edge }}$ region is only affected by a fraction. Furthermore our $f_{\text {edge }}$-method requires quantization of the frequency $f_{a}$ of the surface vibrations in order to determine its amplitude. In our case, this frequency can be obtained by evaluating the dominant frequency in the photodiode's amplitude spectrum or by demodulation techniques which are beyond the scope of this publication. Our proposed $f_{\text {edge }}$ method currently tracks the first order harmonic of the surface vibrations. Coexistence of several higher order harmonics at $-10 \mathrm{~dB}$ with respect to first order harmonics did not pose a problem. As photodiode signal spectra then essentially sum up the effects of more than one harmonic frequency modulation, detecting $f_{\text {edge }}$ of the most dominant harmonic may become more cumbersome. This is the case, when vibration amplitudes $d_{a}$ are too low (i.e. $d_{a}<\lambda$ ) to yield a close estimate of $f_{a}$ or when the vibration frequency does not dominate the photodiode signal. The surface tilt during vibration as well as relaxed precision in the mechanical setup caused the reflected beam in our setup to move laterally along our photodiode surface. Such lateral movement caused the vibration frequency $f_{a}$ to dominate the photodiode spectrum which was beneficial for estimating $f_{a}$. Of course the vibration frequency can also be estimated using an additional detector (e.g. piezo sensor).

\section{Conclusion}

We have introduced a new method for measuring the amplitudes of harmonic surface vibrations well beyond the non-ambiguity range associated with the use of a conventional Michelson interferometer. With relatively simple signal processing it is possible to measure vibration amplitudes at a precision that is comparable to a fringe counting method (FCM) and that is hardly affected by the varying tilt an oscillating surface may exhibit during the reflection of the laser beam. The proposed method allows for monitoring amplitudes which proved to be too small to be reliably measured by FCM. The vibration amplitudes in our experiments were up to $14 \mu \mathrm{m}$ at $79 \mathrm{~Hz}$ vibration frequency. A $192 \mathrm{kHz}$ sampling rate for digitization was applied. The maximum of a $133 \mu \mathrm{m}$ vibration amplitude is the theoretical limit. The prerequisite of this method is that either the vibration has one strong harmonic of which the frequency is known or the major harmonic can be obtained from demodulation of Eq. (3). Or it is enforced to dominate the photodiode spectrum using tilted beam incidence and a smooth surface with directed reflection. The quality control of e.g. loudspeakers, where amplitudes can be much greater than a laser wavelength, may be a potential application for measuring harmonic vibrations with high amplitudes optically. The implementation of more sophisticated algorithms may speed up the calculation and provide even more accurate results. Further investigations have to be done to handle broadband vibration or small amplitudes below the magnitude of the wavelength. Another improvement could be to limit the time span the FFT operates on down to one half of a period $1 / f_{a}$ of the surface oscillation and to shorten that period further by increasing $f_{a}$, if $f_{a}$ is tunable. This then would allow for implementing a fast real time monitoring system for various applications in harmonic vibration detection.

\section{Acknowledgement}

The authors gratefully acknowledge funding of the Erlangen Graduate School in Advanced Optical Technologies (SAOT) by the German Research Foundation (DFG) in the framework of the German excellence initiative.

\section{References}

[1] F. Garoi, D. Apostol, V. Damian, P. Schiopu, Traceable vibration amplitude measurement with a laser interferometer, Rom. Journ. Phys. 14, 369-375 (2010)

[2] J.M. Huntley, H. Saldner, Temporal phaseunwrapping algorithm for automated interferogram analysis, Appl. Opt. 32, 3047-3052 (1993)

[3] W. Shi, Y. Zhu, Y. Yao, Discussion about the DCT/FFT phase-unwrapping algorithm for interferometric applications, Optik 121, 14431449 (2010); doi:10.1016/j.jjleo.2009.02.006

[4] T.K. Gangopadhyay, Non-contact vibration measurement based on an extrinsic Fabry-Perot interferometer implemented using arrays of single-mode fibres, Meas. Sci. Technol. 15, 911917 (2004); doi: 10.1088/0957-0233/15/5/019

[5] H.-J. v. Martens, Interferometric counting methods for measuring displacements in the range $10^{-9}$ to $1 \mathrm{~m}$, Metrologia 24, 163-170 (1987)

[6] R. Dändliker, R. Thalmann, D. Prongué, Twowavelength laser interferometry using superheterodyne detection, Opt. Lett. 13, 339341 (1988)

[7] G.S. Pineda, L.F. Argote, Development of interferometric fringe disappearance method used to calibrate accelerometers, submitted to the SEM Annual Conference on Experimental Mechanics, Portland, Oregon, USA, (2001)

[8] E. Hecht, Optics, Addison Wesley, (2000) 